\title{
Diastropic Dwarfism: a Histochemical and Ultrastructural Study of the Endochondral Growth Plate
}

\author{
WILliAM A. HORTON, DAVID L. RIMOIN, DAVID W. HOLliSTER, AND RUTH SILBERBERG
}

Division of Medical Genetics, UCLA-Harbor General Hospital. Torrance, California, USA

\begin{abstract}
Summary
Chondro-osseous tissue from five patients with diastropic dwarfism was studied by histologic, histochemical, and electron microscopic methods. The major abnormalities observed were: 1) irregular distribution of chondrocytes undergoing degeneration in the resting cartilage; 2 ) abnormal distribution of collagen in the resting cartilage; 3) a spectrum of fibrous matrix lesions in the resting cartilage which ranged from focal areas of aggregated collagen fibrils to large cystic lesions in which intracartilagenous ossification occurred; and 4) shortened, irregular cellular columns within the growth plate which were occasionally disrupted by matrix lesions extending from the resting cartilage. These alterations in chondro-osseous morphology have not been observed in any of the other skeletal dysplasias examined to date and appear to be pathognomonic for this disorder.
\end{abstract}

\section{Speculation}

The basic abnormality in diastropic dwarfism may be a metabolic abnormality in the chondrocyte which predisposes it to cell death or a processing defect in its synthesis of either collagen or proteoglycan. An enzyme deficiency could produce either type of defect and would be consistent with the autosomal recessive inheritance of this disorder.

Diastropic dwarfism is a generalized disorder of cartilage (7, 14). Cartilagenous abnormalities occur in the endochondral growth plate cartilage, resulting in short stature and skeletal deformities; in the joints, producing degenerative arthritis and premature calcification of costal cartilage; in the trachea, resulting in tracheomalacia, and in the ear, leading to "cauliflower ear." In addition, the occurrence of ligamentous laxity in some joints, contractures in others, progressive scoliosis, and club foot suggest that other periarticular structures such as ligaments, joint capsules, etc., may be affected as well (14). The pathogenesis of these changes is poorly understood. Although several reviews of growth plate histopathology in the chondrodystrophies have included descriptions of morphologic abnormalities of cartilage in diastropic dwarfism at both the light and electron microscopic levels $(7-9,12,13)$, these findings have never been reported in detail. Furthermore, a new method has now been developed utilizing plastic embedding to examine the growth plate histochemically (3) which has greatly improved the cellular and matrix detail which we have observed in these specimens. The purpose of this report is to describe the distinctive and diagnostic histochemical and ultrastructural characteristics of chondro-osseous tissue in diastropic dwarfism.

\section{MATERIALS AND METHODS}

$\mathrm{Rib}$ and iliac crest biopsies containing resting and growth plate cartilage and bone were obtained, following appropriate consent, from five individuals, 7- to 14-years-old with well-documented diastropic dwarfism. A portion of the specimen was fixed in $10 \%$ formalin, processed routinely in paraffin, and stained with $\mathrm{H} \& \mathrm{E}$, Masson's Trichrone, and alcian blue/PAS. For electron microscopy, small slices of resting cartilage were fixed in glutaraldehyde and postfixed in osmium tetroxide according to standard techniques. Electron micrographs were taken by an RCA electron microscope model 3F or Phillips model 300.

Another portion of the biopsy specimen was rapidly frozen in liquid nitrogen and stored at $-70^{\circ} \mathrm{C}$. Recently, when plastic embedding methods became feasible (3), specimens from four of these cases were removed from storage for histochemical studies. Before thawing, these specimens were dehydrated in a solution of $70 \%$ ethylene glycol and 30\% formol-calcium (10\% formalin and $2 \%$ calcium acetate) at $-40^{\circ} \mathrm{C}$ for $4-5$ days. Subsequently the temperature was raised to $-10^{\circ} \mathrm{C}$ and the dehydration completed

Table 1. Summary of histopathologic findings in diastropic dwarfism

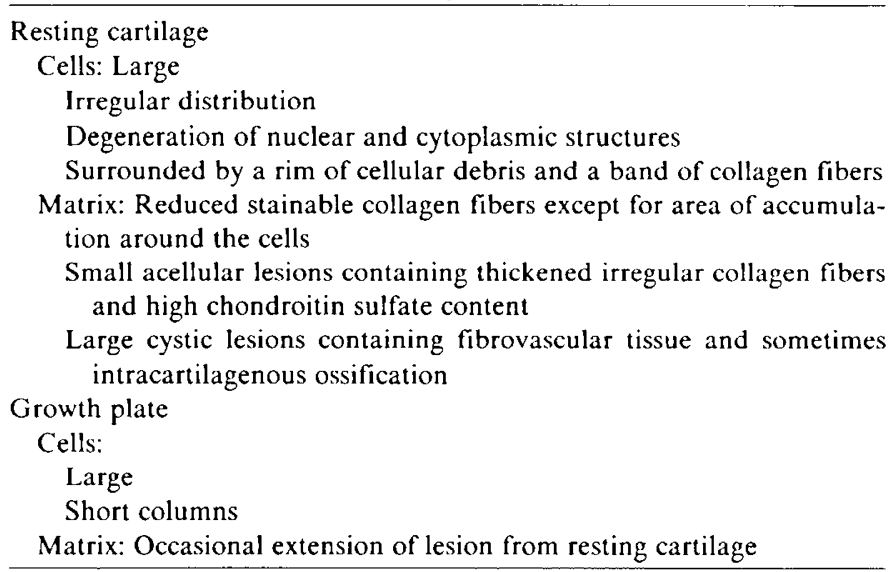

in $100 \%$ ethylene glycol for 1 day. The specimens were then infiltrated with the monomeric glycol methacrylate solution (GMA) (Polysciences) at $4^{\circ} \mathrm{C}$. After four changes of GMA at 12$\mathrm{hr}$ intervals, polymerization was carried out over a 12 -hr period at $4^{\circ} \mathrm{C}$. One micron sections were cut on a Sorvall JB-4 microtome by a $1 / 2$ inch glass knife and were stained with a series of techniques modified for GMA embedded cartilage tissue. The following staining procedures were used: 1) $1 \%$ alcian blue in $0.4 \mathrm{M}$ and $0.9 \mathrm{M} \mathrm{MgCl}_{2}(10,11)$ for $15 \mathrm{~min}$, with and without subsequent digestion by $0.1 \%$ bovine testicular hyaluronidase (4), and counterstained for $1 \mathrm{~min}$ in $1 \times 10^{-3} \mathrm{M}$ basic fuchsin buffered at $\mathrm{pH} 7.4$ (1), to characterize the proteoglycan (protein bound glycosaminoglycan, mucopolysaccharide); 2) a trichrome stain consisting of $0.9 \%$ Biebrich scarlet $/ 0.1 \%$ acetic acid for $5 \mathrm{~min}(6)$ and $0.5 \%$ aniline blue in $0.06 \mathrm{M} \mathrm{HCl}$ for $5 \mathrm{~min}(5)$, combined with the von Kossa $\left(\mathrm{AgNO}_{3}\right)$ method (2) to study calcification; and 3$)$ the van 

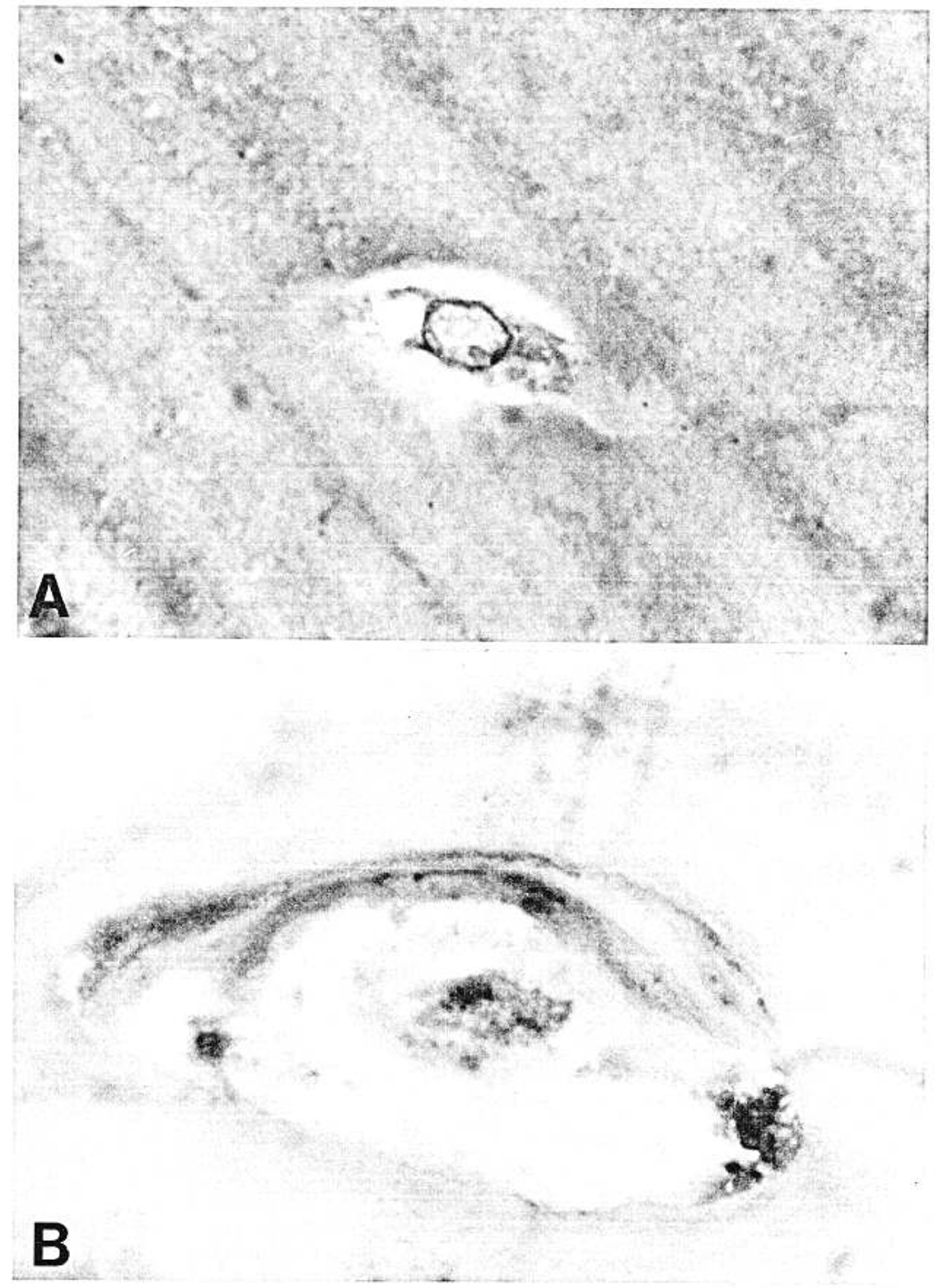

Fig. 1. Resting cartilage. Top, 7-year-old normal female control and bottom, 14-year-old girl with diastropic dwarfism, both Trichrome stain, 1000X GMA. Note the partially degenerated chondrocyte with debris around the periphery of the lacuna in bottom. 




Fig. 2. Methenamine silver staining of resting cartilage. Left, normal 12-year-old control 160X in GMA, and right, diastropic dwarfism, 14-year-old female, 160X in GMA. Note the tendency for the stain to accumulate in the perilacunar area rather than distribute evenly as in the normal control.

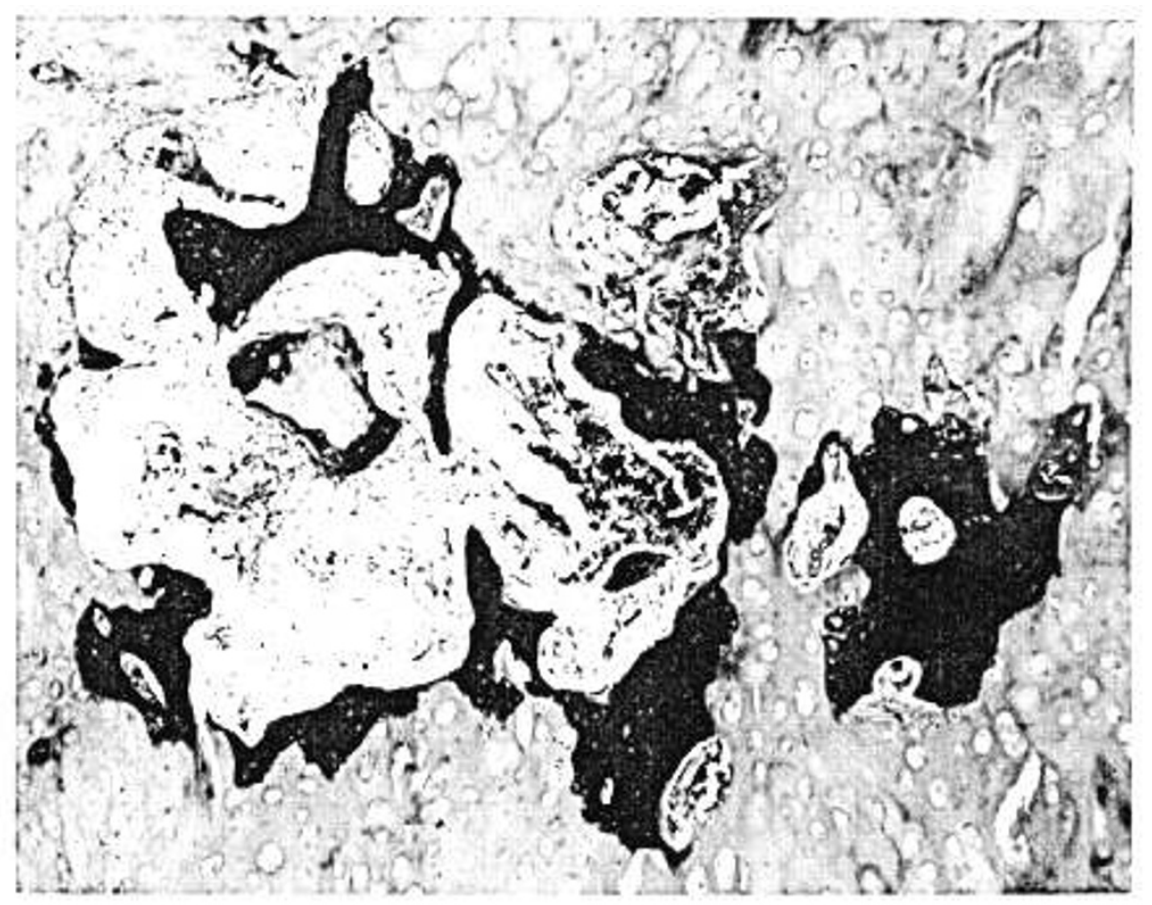

Fig. 3. Resting cartilage from 14-year-old female with diastropic dwarfism. Note the large cyst and adjacent areas of intracartilagenous ossification (black). Masson's trichrome 40X in paraffin. 


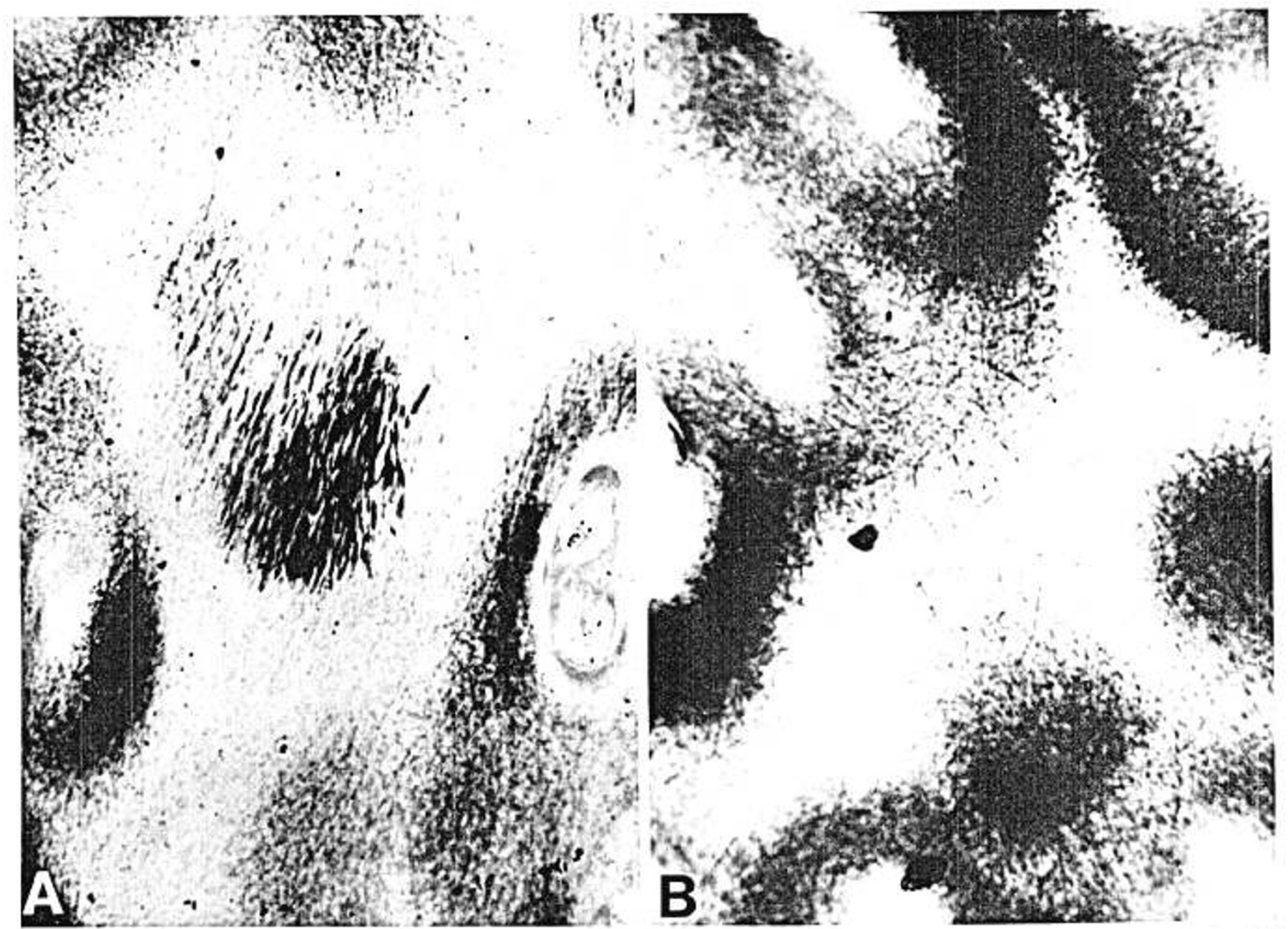

Fig. 4. Methenamine silver staining of fibrous scarring in resting cartilage from 14-year-old female with diastropic dwarfism. Left, typical acellular matrix scar, 500X in GMA. Right, early thickening of collagen fibers in cellular areas, 640X in GMA.

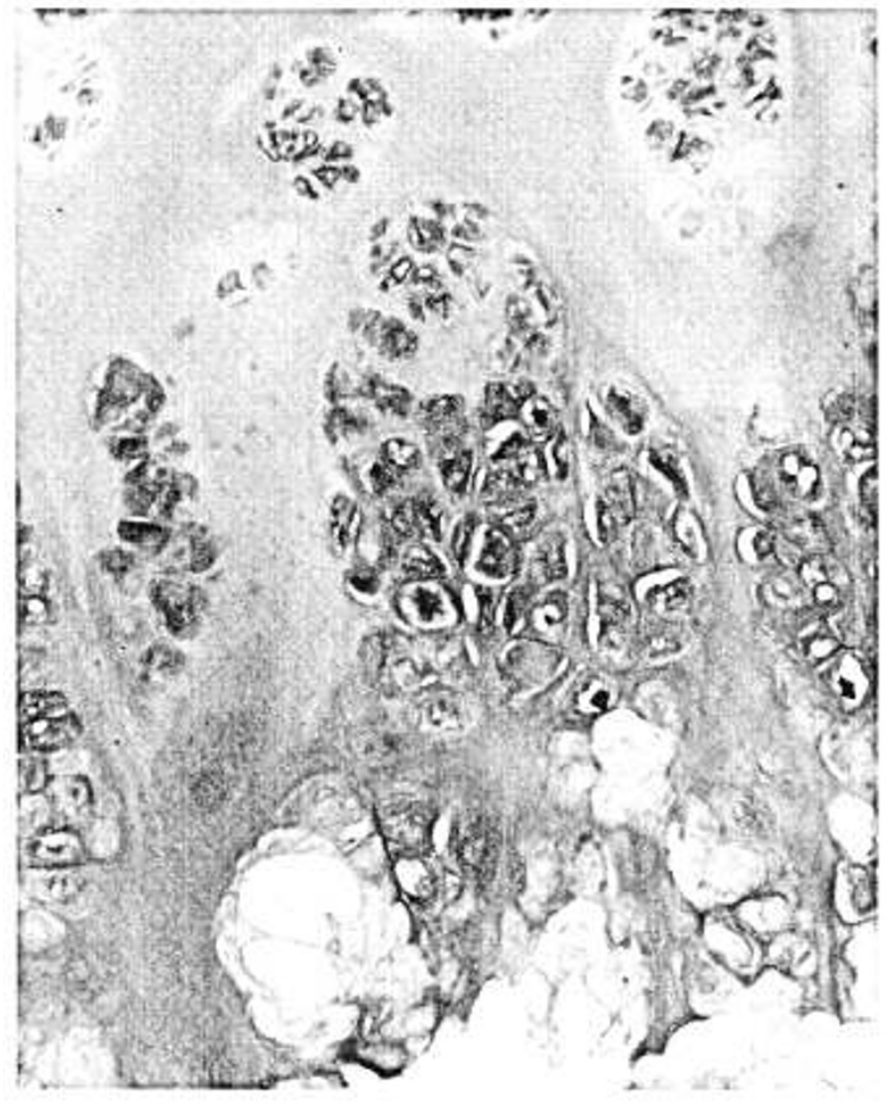

Gieson stain (5) and the silver methenamine technique (6) to evaluate collagen distribution. After consent, cartilage tissue was obtained at time of surgery from seven control individuals who did not suffer from a chondrodystrophy. The procurement was approved by the institutional Human Subjects Committee. The control specimens were processed and studied in a similar fashion.

\section{RESULTS}

Similar histopathologic changes were observed in the rib and iliac crest cartilage from all individuals with diastropic dwarfism who were studied. These are summarized in Table 1. There was no correlation between severity of the histologic lesions and age of the patient, however, the more severely affected patients manifested more pronounced pathologic changes. By light microscopy, striking abnormalities were observed throughout the resting cartilage. Chondrocytes were distributed within the matrix very irregularly and the lacunae often contained three or four chondrocytes, as compared with the one or two chondrocytes seen in the lacunae of control specimens. The lacunae were larger than normal. Individual cells were enlarged and ovoid in shape (Fig. 1). The nuclei were often pyknotic and eccentrically placed. Many cells appeared to be degenerating and a peculiar ring of material was seen around their periphery. The cell cytoplasm, the lacunar area, and halo area surrounding the cell stained intensely with alcian blue in $0.4 \mathrm{M} \mathrm{MgCl}_{2}$ indicating a high glycosaminoglycan content. Furthermore, staining was lost when the $\mathrm{MgCl}_{2}$ concentration was raised to $0.9 \mathrm{M}$ and after digestion by bovine testicular hylauronidase, both of which indicate that the glycosaminoglycan

Fig. 5. Growth plate from 6-year-old female with diastropic dwarfism. Note the short columns. Alcian blue/PAS 160X in paraffin. 


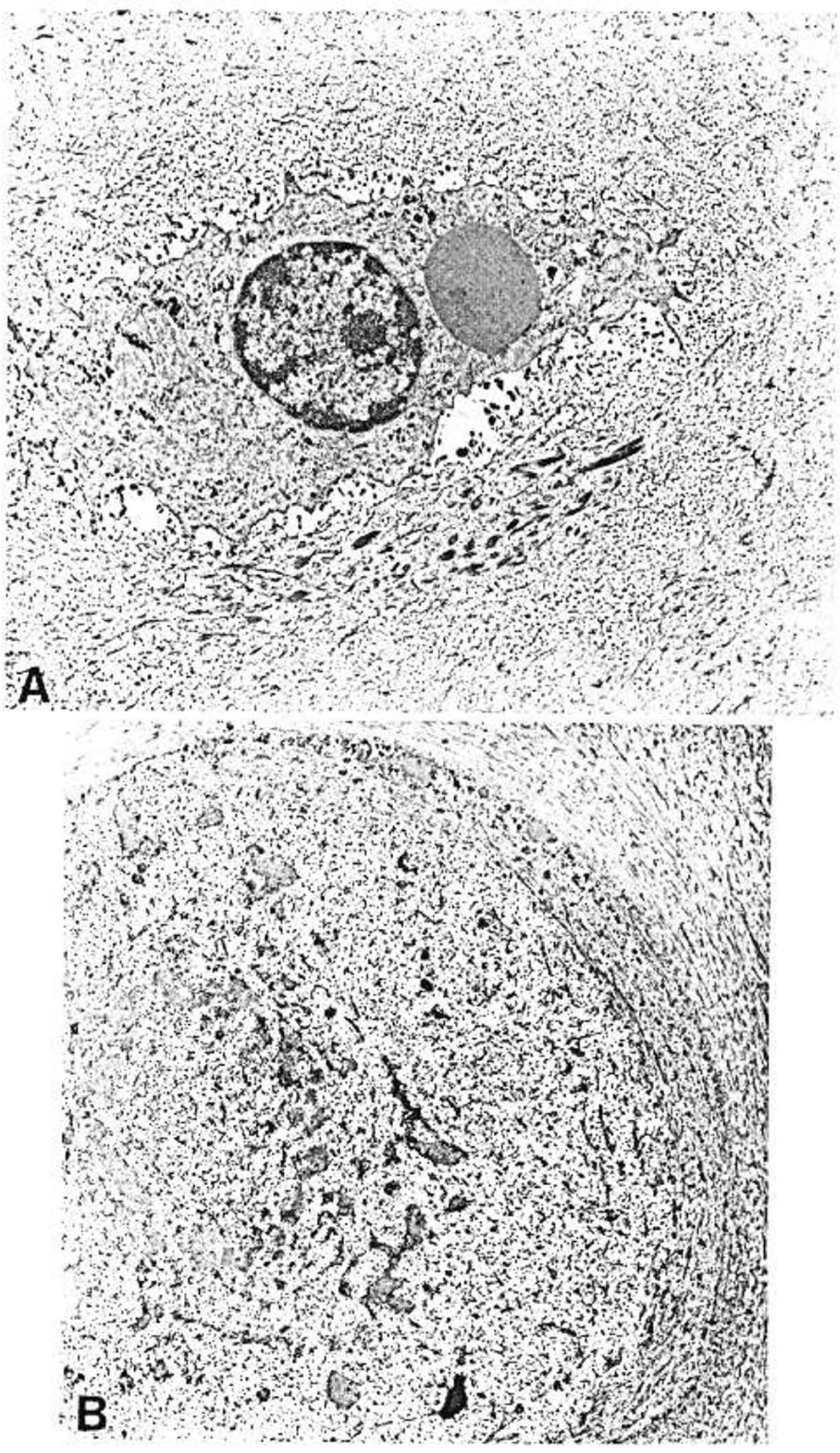

Fig. 6. Electron micrographs of resting chondrocyte from iliac crest of top, 6-year-old female with diastrophic dwarfism. Note the lipid granule in chondrocyte which is surrounded by irregularly scattered and thickened collagen fibrils 18,000X and bottom, 14-year-old female with diastropic dwarfism. Note the circumferentially oriented collagen fibrils surrounding the degenerated chondrocyte $16,500 \mathrm{X}$.

content is predominantly chondroitin sulfate and not keratan sulfate (keratan sulfate continues to stain after both maneuvers).

Abnormal collagen distribution and organization were demonstrated by silver methenamine staining. Normally by this tech- nique, in which silver appears to impregnate collagen fibrils, there is a relatively homogeneous staining of the resting cartilage matrix. The cells themselves and the surrounding extracellular halo area do not stain (Fig. 2, left side). In diastropic dwarfism, however, 
there was poor staining of the matrix except the area immediately surrounding the halo which stained intensely (Fig. 2, right side).

Fibrous lesions within the matrix were present throughout the resting cartilage in areas devoid of cells. These varied from an area the size of a single lacuna to an area which would have contained 20 or more lacunae. In severity they ranged from a minimal fibrous appearance of the matrix to large cystic areas into which fibrovascular ingrowth had occurred and in some cases even intracartilagenous ossification as well (Fig. 3). The smaller lesions not displaying cystic changes were characterized by fibers of varying thickness coursing through lakes of alcian blue positive material which did not stain at $0.9 \mathrm{M} \mathrm{MgCl}_{2}$ or after hylauronidase digestion and were thus composed predominantly of chondroitin sulfate. Alcian blue staining of matrix proteoglycan between cells was not found in control cartilage. Silver methenamine staining revealed that the fibrous component of the lesion was apparently thickened aggregated collagen fibrils (Fig. 4, left side). Slight fiber thickening was observed in areas of the matrix which otherwise appeared normal (Fig. 4, right side), presumably representing the early stage of collagen aggregation. Within the larger lesions containing cystic changes, the alcian blue material was lost and eventually fibers were also lost leaving large open cysts.

Within the growth plate, proliferating and hypertropic chondrocytes tended to be slightly larger than normal. Although somewhat irregular, the column formation and transformation of cartilage into bone appeared normal except for occasional focal areas in which the fibrous matrix lesions from the resting cartilage had extended into the growth plate. In addition, the columns were slightly shorter than normal (Fig. 5). The provisional calcification of cartilage septa, calcification of the metaphyseal bone, and resorption of the calcified spicules progressed normally.

Electron micrographs of the resting cartilage revealed many relatively normal appearing chondrocytes in which intracytoplasmic lipid inclusions were prominent (Fig. 6, top). Other cells showed varying degrees of degeneration and quite often, cellular debris consisting of clumps of ground substance within a finely fibrillar matrix was observed around their periphery (Fig. 6, bottom). This debris was in turn surrounded by bands of circumferentially oriented collagen fibrils which seemed to separate the halo from the remainder of the matrix and corresponded to the intense silver impregnation seen by light microscopy. The matrix itself was generally more fibrillar than normal, and many fibrils were thickened.

\section{DISCUSSION}

Diastropic dwarfism is a unique skeletal dysplasia in that it appears to represent a generalized disorder of cartilage with degenerative changes in aural, laryngeal, and costal cartilage as well as chondro-osseous tissue. Histochemical and ultrastructural analysis of costochondral and iliac crest biopsies from five patients with typical diastropic dwarfism has provided a definition of the spectrum of characteristic and distinctive morphologic alterations in this disorder. As in previous studies $(3,7-9,12,13)$, marked degeneration of resting chondrocytes in cartilage matrix was observed; although some cells appeared normal. In addition, histochemical abnormalities of two of the major components of cartilage matrix, collagen and proteoglycan, were identified. Collagen organization was disturbed; the fibers were thickened and tended to accumulate around chondrocyte lacunae. Normally they are evenly distributed throughout the resting cartilage matrix. In addition, the smaller areas of matrix degeneration contained large amounts of stainable chondroitin sulfate. Similar staining was not found in normal controls suggesting that in this disorder chondroitin sulfate in the matrix is present in larger amounts than normal or that the chemical nature of the matrix has been altered permitting proteoglycan to be stained. This combination of changes has not been seen in any other skeletal dysplasia examined to date and therefore appears to be pathognomonic for the disorder.

The pathogenesis of diastropic dwarfism still remains to be elucidated. The findings of this study show degeneration of both the chondrocytes and the cartilage matrix, but do not indicate the order in which the changes occur. As previously postulated, it is possible that the chondrocyte survival is reduced due to a metabolic derangement, with secondary alteration of the matrix (7). Alternatively, the basic defect might reside in the cartilage matrix. These data do not permit further speculation in this question which can only be answered biochemically. The autosomal recessive inheritance of the disorder does suggest that the basic lesion is a chondrocyte enzyme defect because this cell is responsible for both synthesizing and maintaining the cartilage matrix.

\section{REFERENCES AND NOTES}

1. Bennett, H. S., Wyrick, A. D., Lee, S. W., and McNeil, J. H.: Science and art in preparing tissues embedded in plastic for light microscopy with special reference to glycol methacrylate, glass knives, and simple stains. Stain Technol., 51: $71(1976)$.

2. Culling, C. F. A.: Enzymes. In: Handbook of Histopathological and Histochemical Techniques. p. 470 (Butterworths, Toronto, 1974).

3. Horton, W. A., and Rimoin, D. L.: Histochemical characterization of the growth plate, a new approach to studying the chondrodystrophies. Birth Defects: Original Article Series 14: 81 (1978).

4. Hirshman, A., and McCabe, D. M.: The effect of proteolytic enzymes and hyaluronidase on the intracellular $\beta$ and $\lambda$ metachromatic granules and the matrix of rat epiphyseal cartilage. Anat. Rec., 180 ): 617 (1974).

5. Lillie, R. D. and Fullmer, H. M.: Connective tissue fibers and membranes. In: Histopathologic Technic and Practical Histochemistry. p. 679, 702 (McGraw Hill, New York, 1976).

6. Luna, L. G.: In: Manual of Histologic Staining Methods of the Armed Forces Institute of Pathology. p. 94, 97 (McGraw-Hill, New York, 1968).

7. Rimoin. D. L.: The chondrodystrophies. Adv. Hum. Gen., 5: 1 (1975).

8. Rimoin, D. L., Hollister, D. W., Lachman, R. S., Kaufman, R. L., McAlister, W. H., Rosenthal, R. E., and Hughes, G. N. F.: Histologic studies in the chondrodystrophies. In: D. Bergmsa: Skeletal Dysplasias, Birth Defects: Original Article Series, 10: 274 (1974)

9. Rimoin, D. L., Silberberg. R., and Hollister, D. W.: Chondro-osseous pathology in the chondrodystrophies. Clin. Orthop. Rel. Res., 114: 137 (1976).

10. Scott. J. E., and Dorling. J.: Differential staining of acid glycosaminoglycans (mucopolysaccharides) by alcian blue in salt solutions. Histochemie., 5: 221 (1965).

11. Scott, J. E., Dorling, J., and Stockwell, R. A.: Reversal of protein blocking of basophilia in salt solutions, implications in the localization of polyanions using alcian blue. J. Histochem. Cytochem., 16: 838 (1968).

12. Silberberg, R.: Ultrastructure of cartilage in chondrodystrophies, In: D. Bergsma: Skeletal Dysplasias, Birth Defects: Original Article Series, 10. 306 (1974).

13. Stanescu, V., Stanescu, R., Maroteaux. P.: Etude morphologique et biochemique du cartilage de croissance dans les osteochondrodysplasies. Arch. Franciase Pediatrie., 24: I (1977)

14. Walker, B. A., Scott, C. I., Hall, J. B., Murdoch, J. L., and McKusick, V. A.: Diastrophic dwarfism. Medicine, 51: 41 (1972).

15. The authors thank Ms. Carmen Carpentier for her technical assistance in this study.

16. The present address of Dr. William A. Horton is: Division of Metabolism Endocrinology and Genetics, Department of Medicine, Kansas University Medical Center, Kansas City, Kansas, USA.

17. The present address of Dr. Ruth Silberberg is: Department of Pathology, Hadassah Hebrew University Medical School. Jerusalem. Israel.

18. This research was supported, in part, by United States Public Health Service Research grant HD 11966: Clinical Research Center grant RRO 0425, Graduate Research Training grant GM 07414, and a Research and Birth Defects Center grant from the National Foundation - March of Dimes.

19. Received for publication December 2, 1977

20. Accepted for publication August 10, 1978. 